Pacific

Journal of

Mathematics

A LIOUVILLE-TYPE THEOREM FOR SEMILINEAR ELLIPTIC SYSTEMS VIA MOVING SPHERES

YAJING ZHANG

Volume $236 \quad$ No. 2

June 2008 


\title{
A LIOUVILLE-TYPE THEOREM FOR SEMILINEAR ELLIPTIC SYSTEMS VIA MOVING SPHERES
}

\author{
YAJING ZHANG
}

\begin{abstract}
In this paper, we consider second order semilinear elliptic systems of the form $-\Delta u=a(x) v^{p}$ and $-\Delta v=b(x) u^{q}$ in $\mathbb{R}^{N}$ for $N \geq 3$, where $p, q>0$ and $a, b \in C\left(\mathbb{R}^{N}\right)$. We prove a new Liouville-type theorem for the system under appropriate conditions on the nonlinearity.
\end{abstract}

\section{Introduction}

We consider second order semilinear elliptic systems of the form

$$
\left.\begin{array}{l}
-\Delta u=a(x) v^{p} \\
-\Delta v=b(x) u^{q}
\end{array}\right\} \quad \text { in } \mathbb{R}^{N}
$$

for $N \geq 3$, where $p, q>0$ and $a, b \in C\left(\mathbb{R}^{N}\right)$.

The problem of existence and nonexistence of positive solutions of scalar elliptic equation

$$
-\Delta u=K(x) u^{p} \quad \text { in } \mathbb{R}^{N}
$$

has been investigated by many authors, see [Ding and Ni 1985; Gidas and Spruck 1981; Kusano and Naito 1987; Lin 1998; Ni 1982]. For $p=(N+2) /(N-2)$, we note that (1-2) has a geometric root. Given a smooth positive function $K$ defined on a Riemannian manifold $\left(M, g_{0}\right)$ of dimension $N \geq 3$, we ask whether there exists a metric $g$ conformal to $g_{0}$ such that $K$ is the scalar curvature of the new metric $g$. Let $g=u^{4 /(N-2)} g_{0}$ for some positive function $u$; then the problem reduces to find solutions of the equation

$$
\frac{4(N-1)}{N-2} \Delta_{g_{0}} u-k_{0} u+K u^{(N+2) /(N-2)}=0 \quad \text { in } M,
$$

where $\Delta_{g_{0}}$ is the Laplace-Beltrami operator on $M$ and $k_{0}$ is the scalar curvature of $\left(M, g_{0}\right)$. In the special case that $M=R^{N}$ and $g_{0}$ is the standard metric of $R^{N}$, we have $k_{0} \equiv 0$ and Equation (1-3) reduces to (1-2) with $p=(N+2) /(N-2)$. For

MSC2000: primary 35J60; secondary 35J45.

Keywords: positive solution, Liouville-type theorem, moving spheres.

Research supported by NSFC (grant number 10701051) and the China Postdoctoral Science Foundation. 
more background material and other related problems, we refer to [Ni 1982; Lin $1998]$ and the references therein.

When $a(x)$ and $b(x)$ are positive constants, the system (1-1) becomes the LaneEmden system

$$
\left.\begin{array}{l}
-\Delta u=v^{p} \\
-\Delta v=u^{q}
\end{array}\right\} \quad \text { in } \mathbb{R}^{N}
$$

There are some nonexistence results for positive solutions of the system (1-4); see [Busca and Manásevich 2002; de Figueiredo and Felmer 1994; Mitidieri 1993; 1996; Serrin and Zou 1994; 1996].

Next we turn our attention to system (1-1). As far as the author knows, there are no results that contain nonexistence criteria of positive solutions to (1-1) except for the following result of Mitideri [1996]. Let $a(x)=a(|x|)$ and $b(x)=b(|x|)$.

Theorem. Suppose that $N \geq 3$ and $p, q>1$ and let $a, b \in[0,+\infty) \rightarrow[0,+\infty)$ be functions such that

(i) $a, b \in C[0,+\infty) \bigcap C^{1}(0,+\infty)$ and $a(r), b(r)>0$ for $r>0$;

(ii) $\left(a(r) r^{\delta}\right)^{\prime},\left(b(r) r^{\delta}\right)^{\prime} \geq 0$ and $r>0$ with

$$
\delta=\frac{2(p+1)(q+1)-N(p q-1)}{p+q+2}
$$

(iii) $\lim _{r \rightarrow \infty} a(r) r^{\delta}=\lim _{r \rightarrow \infty} a(r) r^{\delta}=+\infty$.

Then the problem (1-1) has no positive radial solutions.

In this work, we consider nonradial solutions of (1-1), that is, $a(x)$ and $b(x)$ need not be radially symmetric. We generalize Mitidieri's result partially here. Our result is the following.

Theorem 1.1. Suppose that $N \geq 3$ and $p, q>1$ and that the nonnegative functions $a, b$ satisfy

(i) $|x|^{\delta_{1}} a(x)$ and $|x|^{\delta_{2}} b(x)$ are nondecreasing along each ray $\{t \xi: t \geq 0\}$ for any unit vector $\xi$ in $\mathbb{R}^{N}$, where

$$
\delta_{1}=\frac{1}{2}(N+2-p(N-2)) \quad \text { and } \quad \delta_{2}=\frac{1}{2}(N+2-q(N-2)),
$$

(ii) $\lim _{|x| \rightarrow \infty}|x|^{\delta_{1}} a(x)=\lim _{|x| \rightarrow \infty}|x|^{\delta_{2}} b(x)=+\infty$.

Then the system (1-1) has no positive classical solutions.

We can easily obtain a corollary of Theorem 1.1.

Corollary 1.2. If $1<p, q<(N+2) /(N-2)$, then the system (1-4) has no positive classical solutions. 
We note that Figueiredo and Felmer [1994] proved Corollary 1.2, among other things, by using the moving plane method.

In the proof of Theorem 1.1, we use the method of moving spheres, a variant of the method of moving planes. Roughly speaking, we make reflection with respect to spheres instead of planes. The method of moving spheres was used in [Chou and Chu 1993; Padilla 1994; Chen and Li 1995; Li and Zhu 1995; Li and Zhang 2003]. Li and Zhang made significant simplifications and proved some Liouville type theorems for a single equation. The proof of our theorem is along the lines of the works cited above.

The method of moving planes was first introduced by Alexandrov [1958] and then used by several authors: Serrin [1971]; Gidas, Ni and Nirenberg [1979, 1981]; and Berestycki and Nirenberg [1988, 1991]. This method has become a powerful tool in the study of nonlinear partial differential equations.

The paper is organized as follows. In Section 2, we give some lemmas that are used in the proof of Theorem 1.1, which is then proved in Section 3.

\section{Preliminary lemmas}

For $\lambda>0$, consider the Kelvin transformation of $u$ and $v$ for $x \in \mathbb{R}^{N} \backslash\{0\}$ :

$$
u_{\lambda}(x)=\frac{\lambda^{N-2}}{|x|^{N-2}} u\left(\frac{\lambda^{2}}{|x|^{2}} x\right) \quad \text { and } \quad v_{\lambda}(x)=\frac{\lambda^{N-2}}{|x|^{N-2}} v\left(\frac{\lambda^{2}}{|x|^{2}} x\right) .
$$

Our first lemma says that we can initiate the method of moving spheres.

Lemma 2.1. There exists a $\lambda_{0}>0$ such that $u_{\lambda}(x) \leq u(x)$ and $v_{\lambda}(x) \leq v(x)$ for all $0<\lambda<\lambda_{0}$ and $|x| \geq \lambda$.

Proof. Clearly, there exists an $r_{0}>0$ such that

$$
\frac{d}{d r}\left(r^{(N-2) / 2} u(r, \theta)\right)>0 \quad \text { for all } 0<r<r_{0} \text { and } \theta \in S^{N-1} .
$$

Consequently,

$$
u_{\lambda}(x)<u(x) \text { for all } 0<\lambda<|x|<r_{0} .
$$

By the superharmonicity of $u$ and the maximum principle,

$$
u(x) \geq\left(\min _{\partial B_{r_{0}}} u\right) r_{0}^{N-2}|x|^{2-N} \text { for all }|x| \geq r_{0} .
$$

Let

$$
\lambda_{1}=r_{0}\left(\frac{\min _{\partial B_{r_{0}}} u}{\max _{\bar{B}_{r_{0}}} u}\right)^{1 /(N-2)} \leq r_{0} .
$$

Then for every $0<\lambda<\lambda_{1}$ and $|x| \geq r_{0}$, we have

$$
u_{\lambda}(x) \leq \frac{\lambda_{1}^{N-2}}{|x|^{N-2}} \max _{\bar{B}_{r_{0}}} u \leq \frac{r_{0}^{N-2} \min _{\partial B_{r_{0}}} u}{|x|^{N-2}} \leq u(x) .
$$


Similarly, there exists $\lambda_{2}>0$ such that for every $0<\lambda<\lambda_{2}$, we have

$$
v_{\lambda}(x) \leq v(x) \text { for }|x| \geq \lambda .
$$

We choose $\lambda_{0}=\min \left\{\lambda_{1}, \lambda_{2}\right\}$. Thus, for every $0<\lambda<\lambda_{0}$,

$$
u_{\lambda}(x) \leq u(x) \quad \text { and } \quad v_{\lambda}(x) \leq v(x) \quad \text { for }|x| \geq \lambda .
$$

Set

$\bar{\lambda}=\sup \left\{\mu>0: u_{\lambda}(x) \leq u(x)\right.$ and $v_{\lambda}(x) \leq v(x)$ for all $|x| \geq \lambda$ and $\left.0<\lambda \leq \mu\right\}$.

By Lemma 2.1, $\bar{\lambda}$ is well defined, and $0<\bar{\lambda} \leq+\infty$.

Lemma 2.2. If $\bar{\lambda}<+\infty$, then $u_{\bar{\lambda}}(x) \equiv u(x)$ and $v_{\bar{\lambda}}(x) \equiv v(x)$ in $\mathbb{R}^{N} \backslash\{0\}$.

Proof. Let $\Sigma_{\lambda}=\{x:|x|>\lambda\}$. Clearly it suffices to show

$$
u_{\bar{\lambda}} \equiv u \quad \text { and } \quad v_{\bar{\lambda}} \equiv v \quad \text { in } \Sigma_{\bar{\lambda}} .
$$

We first prove $u_{\bar{\lambda}} \equiv u$. We prove it by contradiction. Supposing $u_{\bar{\lambda}} \not \equiv u$ in $\Sigma_{\bar{\lambda}}$, we know from the definition of $\bar{\lambda}$ that

$$
u_{\bar{\lambda}} \leq u \quad \text { and } \quad v_{\bar{\lambda}} \leq v \quad \text { in } \Sigma_{\bar{\lambda}} .
$$

From (1-1), a direct calculation yields

$$
-\Delta u_{\lambda}=\left(\frac{\lambda}{|x|}\right)^{N+2-p(N-2)} a\left(\frac{\lambda^{2}}{|x|^{2}} x\right) v_{\lambda}^{p} .
$$

Thus, by condition (i) of Theorem 1.1, we have

$$
\begin{aligned}
-\Delta\left(u-u_{\bar{\lambda}}\right) & =a(x) v^{p}-\left(\frac{\bar{\lambda}}{|x|}\right)^{N+2-p(N-2)} a\left(\frac{\bar{\lambda}^{2}}{|x|^{2}} x\right) v_{\bar{\lambda}}^{p} \\
& =|x|^{-\delta_{1}}\left(|x|^{\delta_{1}} a(x) v^{p}-|x|^{\delta_{1}}\left(\frac{\bar{\lambda}}{|x|}\right)^{N+2-p(N-2)} a\left(\frac{\bar{\lambda}^{2}}{|x|^{2}} x\right) v_{\bar{\lambda}}^{p}\right) \\
& =|x|^{-\delta_{1}}\left(|x|^{\delta_{1}} a(x) v^{p}-\left(\frac{\bar{\lambda}^{2}}{|x|}\right)^{\delta_{1}} a\left(\frac{\bar{\lambda}^{2}}{|x|^{2}} x\right) v_{\bar{\lambda}}^{p}\right) \\
& \geq 0 \quad \text { in } \Sigma_{\bar{\lambda}} .
\end{aligned}
$$

By the maximum principle, $u-u_{\bar{\lambda}}>0$ in $\Sigma_{\bar{\lambda}}$. Thus, by the Hopf lemma and the compactness of $\partial B_{\bar{\lambda}}$, there exists a positive constant $b$ such that

$$
\left.\frac{d}{d r}\left(u-u_{\bar{\lambda}}\right)\right|_{\partial B_{\bar{\lambda}}}>b>0 .
$$

By the continuity of $\nabla u$, there exists $R>\bar{\lambda}$ such that

$$
\frac{d}{d r}\left(u-u_{\lambda}\right)>\frac{b}{2}>0 \text { for } \bar{\lambda} \leq \lambda \leq R \text { and } \lambda \leq r \leq R .
$$


Consequently, since $u-u_{\lambda} \equiv 0$ on $\partial B_{\lambda}$,

$$
u(x)>u_{\lambda}(x) \text { for } \bar{\lambda} \leq \lambda \leq R \text { and } \lambda \leq|x| \leq R .
$$

Set $c=\min _{\partial B_{R}}\left(u-u_{\bar{\lambda}}\right)>0$. It follows from the superharmonicity of $u-u_{\bar{\lambda}}$ that $u-u_{\bar{\lambda}} \geq c R^{N-2} /|x|^{N-2}$ for $|x| \geq R$. Therefore

$$
u-u_{\lambda} \geq \frac{c R^{N-2}}{|x|^{N-2}}-\left(u_{\lambda}-u_{\bar{\lambda}}\right) \quad \text { for }|x| \geq R .
$$

By the uniform continuity of $u$ on $\bar{B}_{R}$, there exists an $\varepsilon \in(0, R-\bar{\lambda})$ such that for all $\bar{\lambda} \leq \lambda \leq \bar{\lambda}+\varepsilon$,

$$
\left|\lambda^{N-2} u\left(\frac{\lambda^{2} x}{|x|^{2}}\right)-\bar{\lambda}^{N-2} u\left(\frac{\bar{\lambda}^{2} x}{|x|^{2}}\right)\right|<\frac{c R^{N-2}}{2} \text { for }|x| \geq R .
$$

It follows from (2-2) and the above inequality that

$$
u(x)-u_{\lambda}(x)>0 \text { for } \bar{\lambda} \leq \lambda \leq \bar{\lambda}+\varepsilon \text { and }|x| \geq R .
$$

Thus, (2-1) and (2-2) are in contradiction with the definition of $\bar{\lambda}$. Similarly, we can prove that $v_{\bar{\lambda}} \equiv v$ in $\Sigma_{\bar{\lambda}}$.

Lemma 2.3. $\quad \bar{\lambda}<+\infty$.

Proof. Suppose by way of contradiction that $\bar{\lambda}=+\infty$. By the definition of $\bar{\lambda}$,

$$
u(x) \geq u_{\lambda}(x) \text { for }|x| \geq \lambda \text { for all } \lambda>0 .
$$

Set $|x|=\lambda^{2}$. Then, from (2-3),

$$
u(x) \geq \lambda^{-(N-2)} u\left(x / \lambda^{2}\right) \geq c_{1}|x|^{-(N-2) / 2} \text { for }|x| \geq 1,
$$

where $c_{1}=\min _{\partial B_{1}} u(x)$. Similarly, we have

$$
v(x) \geq c_{2}|x|^{-(N-2) / 2} \quad \text { for }|x| \geq 1,
$$

where $c_{2}=\min _{\partial B_{1}} v(x)$. Rewrite system (1-1) into

$$
\Delta u+\tilde{a}(x) v=0 \quad \text { and } \quad \Delta v+\tilde{b}(x) u=0,
$$

where $\tilde{a}(x)=a(x) v^{p-1}$ and $\tilde{b}(x)=b(x) u^{q-1}$. For any $M>0$, by condition (ii) of Theorem 1.1, there exists $R_{0} \geq 1$ such that

$$
\tilde{a}(x)|x|^{2} \geq M c_{2}^{1-p}|x|^{2-\delta_{1}}\left(c_{2}|x|^{-(N-2) / 2}\right)^{p-1}=M \quad \text { for }|x| \geq R_{0} .
$$

Thus

$$
\lim _{|x| \rightarrow+\infty} \tilde{a}(x)|x|^{2}=+\infty
$$


Similarly, we have

$$
\lim _{|x| \rightarrow+\infty} \tilde{b}(x)|x|^{2}=+\infty .
$$

For $|y| \leq 1 / 2$, set $\tilde{u}(y)=u(x+|x| y)$ and $\tilde{v}(y)=v(x+|x| y)$. Then $\tilde{u}$ and $\tilde{v}$ satisfy

$$
\left.\begin{array}{l}
\Delta \tilde{u}+|x|^{2} \tilde{a}(x+|x| y) \tilde{v}=0 \\
\Delta \tilde{v}+|x|^{2} \tilde{b}(x+|x| y) \tilde{u}=0
\end{array}\right\} \quad \text { in } B_{1 / 2} .
$$

in $B_{1 / 2}$. Set $f(x)=\inf _{|y| \leq 1 / 2}|x|^{2} \tilde{a}(x+|x| y)$ and $g(x)=\inf _{|y| \leq 1 / 2}|x|^{2} \tilde{b}(x+|x| y)$. From (2-6),

$$
\left.\begin{array}{l}
-\Delta \tilde{u} \geq f(x) \tilde{v} \\
-\Delta \tilde{v} \geq g(x) \tilde{u}
\end{array}\right\} \quad \text { in } B_{1 / 2} .
$$

Let $\phi \in H_{0}^{1}\left(B_{1 / 2}\right)$ be the positive eigenfunction corresponding to the first eigenvalue $\lambda_{1}$ of $\left(-\Delta, H_{0}^{1}\left(B_{1 / 2}\right)\right)$. Multiplying both sides of the first inequality in (2-7) by $\phi$ and integrating the obtained inequality over $B_{1 / 2}$, we have

$$
\begin{aligned}
f(x) \int_{B_{1 / 2}} \tilde{v} \phi d y & \leq \int_{B_{1 / 2}}-\Delta \tilde{u} \cdot \phi d y \\
& \leq \int_{B_{1 / 2}}-\Delta \phi \cdot \tilde{u} d y=\lambda_{1} \int_{B_{1 / 2}} \tilde{u} \phi d y .
\end{aligned}
$$

Similarly, by the second inequality in (2-7), we obtain

$$
g(x) \int_{B_{1 / 2}} \tilde{u} \phi d y \leq \lambda_{1} \int_{B_{1 / 2}} \tilde{v} \phi d y .
$$

From (2-8) and (2-9), $f(x) g(x) \leq \lambda_{1}^{2}$. However, by (2-4) and (2-5),

$$
\lim _{|x| \rightarrow+\infty}|x|^{2} \tilde{a}(x+|x| y)=\lim _{|x| \rightarrow+\infty}|x|^{2} \tilde{b}(x+|x| y)=+\infty,
$$

uniformly for $|y| \leq 1 / 2$. This yields a contradiction.

\section{Proof of Theorem 1.1}

By Lemmas 2.2 and 2.3, we have

$$
u_{\bar{\lambda}}(x) \equiv u(x) \quad \text { and } \quad v_{\bar{\lambda}}(x) \equiv v(x) \quad \text { in } \mathbb{R}^{N} \backslash\{0\} .
$$

Since

$$
\begin{aligned}
& -\Delta u_{\lambda}=\left(\frac{\lambda}{|x|}\right)^{N+2-p(N-2)} a\left(\frac{\lambda^{2}}{|x|^{2}} x\right) v_{\lambda}^{p}, \\
& -\Delta v_{\lambda}=\left(\frac{\lambda}{|x|}\right)^{N+2-q(N-2)} b\left(\frac{\lambda^{2}}{|x|^{2}} x\right) u_{\lambda}^{q},
\end{aligned}
$$


we have by $(1-1)$

$$
\left(\frac{\bar{\lambda}}{|x|}\right)^{N+2-p(N-2)} a\left(\frac{\bar{\lambda}^{2}}{|x|^{2}} x\right) \equiv a(x) \quad \text { in } \mathbb{R}^{N} \backslash\{0\},
$$

that is,

$$
\left(\frac{\bar{\lambda}^{2}}{|x|}\right)^{\delta_{1}} a\left(\frac{\bar{\lambda}^{2}}{|x|^{2}} x\right) \equiv|x|^{\delta_{1}} a(x) \text { in } \mathbb{R}^{N} \backslash\{0\} .
$$

For large $|x|$, we have, by condition (ii) of Theorem 1.1,

$$
\left(\frac{\bar{\lambda}^{2}}{|x|}\right)^{\delta_{1}} a\left(\frac{\bar{\lambda}^{2}}{|x|^{2}} x\right)<|x|^{\delta_{1}} a(x)
$$

which contradicts (3-1).

\section{References}

[Aleksandrov 1958] A. D. Aleksandrov, "Uniqueness theorems for surfaces in the large, V", Vestnik Leningrad. Univ. 13:19 (1958), 5-8. In Russian; translated in Amer. Math. Soc. Transl. Ser. 21 (1962) 412-416. MR 21 \#909 Zbl 0113.16101

[Berestycki and Nirenberg 1988] H. Berestycki and L. Nirenberg, "Monotonicity, symmetry and antisymmetry of solutions of semilinear elliptic equations", J. Geom. Phys. 5:2 (1988), 237-275. MR 91a:35060 Zbl 0698.35031

[Berestycki and Nirenberg 1991] H. Berestycki and L. Nirenberg, "On the method of moving planes and the sliding method", Bol. Soc. Brasil. Mat. (N.S.) 22:1 (1991), 1-37. MR 93a:35048 Zbl 0784. 35025

[Busca and Manásevich 2002] J. Busca and R. Manásevich, "A Liouville-type theorem for LaneEmden systems", Indiana Univ. Math. J. 51:1 (2002), 37-51. MR 2003d:35080 Zbl 1033.35032

[Chen and Li 1995] W. X. Chen and C. Li, "A necessary and sufficient condition for the Nirenberg problem”, Comm. Pure Appl. Math. 48:6 (1995), 657-667. MR 96j:35056 Zbl 0830.35034

[Chou and Chu 1993] K. S. Chou and C. W. Chu, "On the best constant for a weighted SobolevHardy inequality”, J. London Math. Soc. (2) 48:1 (1993), 137-151. MR 94h:46052 Zbl 0739.26013

[Ding and Ni 1985] W. Y. Ding and W.-M. Ni, "On the elliptic equation $\Delta u+K u^{(n+2) /(n-2)}=0$ and related topics”, Duke Math. J. 52:2 (1985), 485-506. MR 86k:35040 Zbl 0592.35048

[de Figueiredo and Felmer 1994] D. G. de Figueiredo and P. L. Felmer, "A Liouville-type theorem for elliptic systems”, Ann. Scuola Norm. Sup. Pisa Cl. Sci. (4) 21 (1994), 387-397. MR 95m:35009 Zbl 0820.35042

[Gidas and Spruck 1981] B. Gidas and J. Spruck, "Global and local behavior of positive solutions of nonlinear elliptic equations", Comm. Pure Appl. Math. 34:4 (1981), 525-598. MR 83f:35045 Zbl 0465.35003

[Gidas et al. 1979] B. Gidas, W. M. Ni, and L. Nirenberg, "Symmetry and related properties via the maximum principle”, Comm. Math. Phys. 68:3 (1979), 209-243. MR 80h:35043 Zbl 0425.35020

[Gidas et al. 1981] B. Gidas, W. M. Ni, and L. Nirenberg, "Symmetry of positive solutions of nonlinear elliptic equations in $\mathbb{R}^{n}$ ", pp. 369-402 in Mathematical analysis and applications, Part A, edited by L. Nachbin, Adv. in Math. Suppl. Stud. 7, Academic Press, New York, 1981. MR 84a:35083 Zbl 0469.35052 
[Kusano and Naito 1987] T. Kusano and M. Naito, "Oscillation theory of entire solutions of second order superlinear elliptic equations”, Funkcial. Ekvac. 30:2-3 (1987), 269-282. MR 89a:35019 Zbl 0656.35040

[Li and Zhang 2003] Y. Li and L. Zhang, "Liouville-type theorems and Harnack-type inequalities for semilinear elliptic equations", J. Anal. Math. 90 (2003), 27-87. MR 2004i:35118

[Li and Zhu 1995] Y. Li and M. Zhu, "Uniqueness theorems through the method of moving spheres", Duke Math. J. 80:2 (1995), 383-417. MR 96k:35061 Zbl 0846.35050

[Lin 1998] C-S. Lin, "Liouville-type theorems for semilinear elliptic equations involving the Sobolev exponent”, Math. Z. 228:4 (1998), 723-744. MR 99f:35050 Zbl 0915.35036

[Mitidieri 1993] E. Mitidieri, "A Rellich type identity and applications", Comm. Partial Differential Equations 18:1-2 (1993), 125-151. MR 94c:26016 Zbl 0816.35027

[Mitidieri 1996] E. Mitidieri, "Nonexistence of positive solutions of semilinear elliptic systems in $\mathbf{R}^{N}$,, Differential Integral Equations 9:3 (1996), 465-479. MR 96m:35087 Zbl 0848.35034

[Ni 1982] W. M. Ni, "On the elliptic equation $\Delta u+K(x) u^{(n+2) /(n-2)}=0$, its generalizations, and applications in geometry", Indiana Univ. Math. J. 31:4 (1982), 493-529. MR 84e:35049 Zbl 0496.35036

[Padilla 1994] P. Padilla, On some nonlinear elliptic equations, Thesis, New York University, 1994.

[Serrin 1971] J. Serrin, "A symmetry problem in potential theory”, Arch. Rational Mech. Anal. 43 (1971), 304-318. MR 48 \#11545 Zbl 0222.31007

[Serrin and Zou 1994] J. Serrin and H. Zou, "Non-existence of positive solutions of semilinear elliptic systems", pp. 55-68 in A tribute to Ilya Bakelman (College Station, TX, 1993), edited by I. R. Bakelman et al., Discourses Math. Appl. 3, Texas A \& M Univ., 1994. MR 97k:35065 Zbl 0900.35121

[Serrin and Zou 1996] J. Serrin and H. Zou, "Non-existence of positive solutions of Lane-Emden systems”, Differential Integral Equations 9:4 (1996), 635-653. MR 97f:35056 Zbl 0868.35032

Received September 21, 2007. Revised March 16, 2008.

\author{
YAJING ZHANG \\ SCHOOL OF MATHEMATICAL SCIENCES \\ SHANXI UNIVERSITY \\ TAIYUAN 030006 \\ CHINA
}

zhangyj@sxu.edu.cn 